\title{
GRAPHS WITH 4-RAINBOW INDEX 3 AND $n-1$
}

\author{
Xueliang Li $^{1}$, Ingo Schiermeyer ${ }^{2}$ \\ KANG YANG ${ }^{1}$ AND YAN ZHAO ${ }^{1}$ \\ ${ }^{1}$ Center for Combinatorics and LPMC-TJKLC \\ Nankai University \\ Tianjin 300071, China \\ ${ }^{2}$ Institut für Diskrete Mathematik und Algebra \\ Technische Universität Bergakademie Frieiberg \\ 09596 Freiberg, Germany \\ e-mail: lxl@nankai.edu.cn \\ Ingo.Schiermeyer@tu-freiberg.de \\ yangkang@mail.nankai.edu.cn \\ zhaoyan2010@mail.nankai.edu.cn
}

\begin{abstract}
Let $G$ be a nontrivial connected graph with an edge-coloring $c: E(G) \rightarrow$ $\{1,2, \ldots, q\}, q \in \mathbb{N}$, where adjacent edges may be colored the same. A tree $T$ in $G$ is called a rainbow tree if no two edges of $T$ receive the same color. For a vertex set $S \subseteq V(G)$, a tree that connects $S$ in $G$ is called an $S$-tree. The minimum number of colors that are needed in an edge-coloring of $G$ such that there is a rainbow $S$-tree for every set $S$ of $k$ vertices of $V(G)$ is called the $k$-rainbow index of $G$, denoted by $r x_{k}(G)$. Notice that a lower bound and an upper bound of the $k$-rainbow index of a graph with order $n$ is $k-1$ and $n-1$, respectively. Chartrand et al. got that the $k$-rainbow index of a tree with order $n$ is $n-1$ and the $k$-rainbow index of a unicyclic graph with order $n$ is $n-1$ or $n-2$. Li and Sun raised the open problem of characterizing the graphs of order $n$ with $r x_{k}(G)=n-1$ for $k \geq 3$. In early papers we characterized the graphs of order $n$ with 3 -rainbow index 2 and $n-1$. In this paper, we focus on $k=4$, and characterize the graphs of order $n$ with 4-rainbow index 3 and $n-1$, respectively.
\end{abstract}

Keywords: rainbow $S$-tree, $k$-rainbow index.

2010 Mathematics Subject Classification: 05C05, 05C15, $05 \mathrm{C} 75$.

\section{REFERENCES}


[1] J.A. Bondy and U.S.R. Murty, Graph Theory (GTM 244, Springer, 2008).

[2] Q. Cai, X. Li and J. Song, Solutions to conjectures on the $(k, \ell)$-rainbow index of complete graphs, Networks 62 (2013) 220-224. doi:10.1002/net.21513

[3] Y. Caro, A. Lev, Y. Roditty, Zs. Tuza and R. Yuster, On rainbow connection, Electron. J. Combin. 15 (2008) R57.

[4] G. Chartrand, G.L. Johns, K.A. McKeon and P. Zhang, Rainbow connection in graphs, Math. Bohem. 133 (2008) 85-98.

[5] G. Chartrand, G.L. Johns, K.A. McKeon and P. Zhang, The rainbow connectivity of a graph, Networks 54 (2009) 75-81. doi:10.1002/net.20296

[6] G. Chartrand, S.F. Kappor, L. Lesniak and D.R. Lick, Generalized connectivity in graphs, Bull. Bombay Math. Colloq 2 (1984) 1-6.

[7] G. Chartrand, F. Okamoto and P. Zhang, Rainbow trees in graphs and generalized connectivity, Networks 55 (2010) 360-367. doi:10.1002/net.20399

[8] L. Chen, X. Li, K. Yang and Y. Zhao, The 3-rainbow index of a graph, Discuss. Math. Graph Theory 35 (2015) 81-94. doi:10.7151/dmgt.1780

[9] P. Erdős and A. Gyárfás, A variant of the classical Ramsey problem, Combinatorica 17 (1997) 459-467. doi:10.1007/BF01195000

[10] X. Li and Y. Sun, Rainbow Connections of Graphs (Springer Briefs in Math., Springer, New York, 2012).

[11] X. Li, Y. Shi and Y. Sun, Rainbow connections of graphs: A survey, Graphs Combin. 29 (2013) $1-38$. doi:10.1007/s00373-012-1243-2

[12] X. Li, I. Schiermeyer, K. Yang and Y. Zhao, Graphs with 3-rainbow index $n-1$ and $n-2$, Discuss. Math. Graph Theory 35 (2015) 105-120. doi: $10.7151 /$ dmgt.1783

Received 14 January 2014

Revised 22 May 2014

Accepted 16 June 2014 